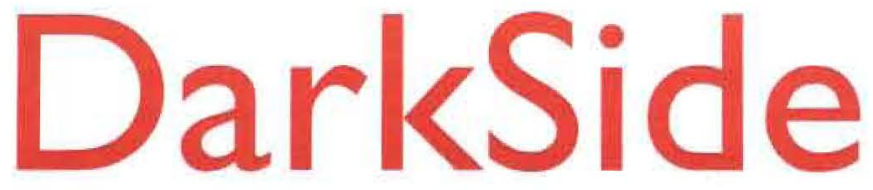

\title{
A Depleted Argon Dark Matter Search
}

Fermilab

October 192009
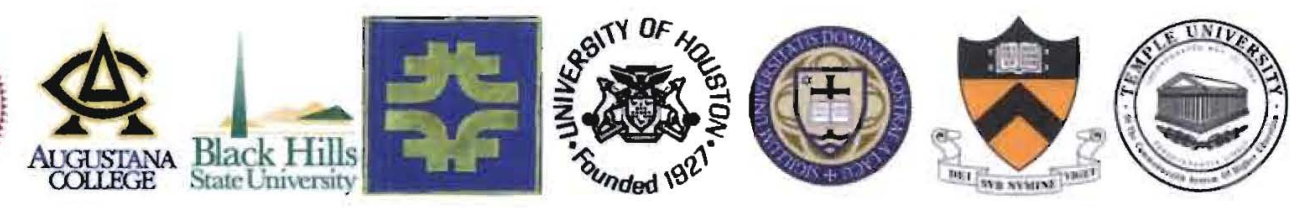

UMass Amherst

Arizona State University

Augustana College

Black Hills State University

Fermilab

University of Houston

University of Notre Dame

Princeton University

Temple University

UCLA 
Project Summary

The existence of dark matter is known from gravitational effects, but its nature remains a deep mystery. One possibility motivated by other considerations in elementary particle physics is that dark matter consists of undiscovered elementary particles. Axions and Weakly Interacting Massive Particles (WIMPs) are two possibilities. Evidence for new particles that could constitute WIMP dark matter may come from upcoming experiments at the Large Hadron Collider at CERN or from sensitive astronomical instruments that detect radiation produced by WIMP.WIMP annihilations in galaxy halos. The thermal motion of the WIMPS comprising the dark matter halo surrounding the galaxy and the earth should result in WIMP-nuclear collisions of sufficient energy to be observable by sensitive laboratory apparatus

The goal of this proposal is to develop and deploy a liquid argon detector that has high sensitivity for direct detection of WIMP collisions. Liquid argon is a promising medium for WIMP detection due to its efficient conversion of energy from WIMP induced nuclear recoils into both ionization and scintillation. In a Time Projection Chamber (TPC), scintillation and ionization can be independently detected and spatially resolved through large volumes of liquid. The relative size and time dependence of these signals permits discrimination of nuclear recoils from background events.

For events resulting in a recolling electron, such as beta or gamma events, the low density of electron-ion pairs results in less recombination and therefore less scintillation and more free electrons, as compared to a nuclear recoil track of high ionization density. Therefore the ratio of ionization to scintillation thus allows separation of background events due to electron recoils from those due to nuclear recols. The difference in ionization density between nuclear and electron recoils also produces a significant difference in the time profile of the scintillation light. Scintillation in argon has a long-lived $(\sim 1.6 \mu s)$ triplet state, and a shortwived ( $\sim 6$ ns) singlet state. The long-lived state is found to be quenched in tracks with high ionization density. Thus, electron recoils have longer scintillation duration compared to nuclear recoils, providing a very powerful "pulse shape discrimination" (PSD) between electron backgrounds and nuclear-recoll signal.

By exploiting these methods, the WARP-3.2 argon TPC experiment achieved competitive sensitivity to WIMPs in 2007, reporting a cross section limit of $10^{-47} \mathrm{~cm}^{2}$. The Princeton group is participating in the WARP-140 experiment, now being commissioned at LNGS, with an anticipated sensitivity of $1 \times 10^{-44} \mathrm{~cm}^{2}$.

The argon detector proposed here, DARKSIDE-50, builds on past experience and introduces innovative features that will allow it to operate in a background-free mode for more than three years and thereby achieve a significant science result in spite of its relatively small size. At the same time this detector will serve as a prototype for a future multi-ton detector. The main innovations introduced in this proposal are:

- Underground argon depleted in radioactive ${ }^{39} \mathrm{Ar}$;

- Low background, high-quantum-efficiency QuPID photo detectors;

- A compact high-efficiency external veto for neutrons.

These innovations, together with the powerful two-parameter background rejection features of argon will result in a detector of unprecedented background-free performance. With a mass of $50 \mathrm{~kg}(33 \mathrm{~kg}$ fiducial) and three years of operation, the DARKSIDE-50 detector will reach a cross-section sensitivity of $1.5 \times 10^{-45} \mathrm{~cm}^{2}$ for WIMP-nucleon scattering, an improvement of more than a factor of 10 compared to published results on spin independent cross sections for WIMPs of $100 \mathrm{GeV}$ mass, and competitive with the projected sensitivity of WARP140, SUPER-CDMS at SNOLab. LUX-350, and XENON-100 detectors. DARKSIDE-50 will benefit from the facility for extraction and refinement of depleted argon, independently funded by US National Science Foundation (NSF) through a grant to Princeton University. DARKSDDE-50 will also benefit from the engineering work being carried out by a consortium of the DARKSIDE and XENON collaborations known as "Multi-ton Argon and Xenon TPCs" (MAX for short), supported by the NSF under the Deep Underground Science and Engineering Laboratory (DUSEL) S4 program. DARKSIDE-50 will in turn allow validation of some technical choices for the MAX design.

INTELLECTUAL MERIT: DARKSIDE-50 will either detect WIMP dark matter or exclude a substantial fraction of the favored parameter space. The proposed activity will also lay the technical foundation for the next generation of larger noble liquid detectors, designed to advance our knowledge of elementary particles and cosmology in fundamental ways by detection of extremely weak, rare, low energy phenomena.

BROADER IMPACT: The proposed activity will advance the development of astroparticle physics and its scientific and educational mission in a variety of ways: (1) it will offer an excellent opportunity for the training of students, who will have a chance to contribute to the success of a cutting edge project in fundamental science and advanced engineering; (2) it will benefit society by developing techniques that could find application in areas ranging from national security to medical imaging; (3) it will support continued development of successful E\&O programs such as the Princeton-Abruzzo-South Dakota summer school for high school students. 

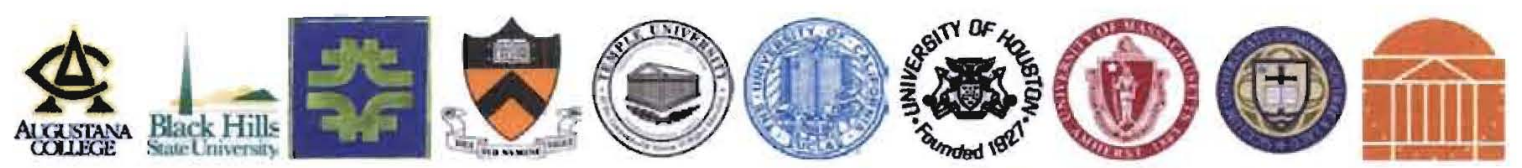

Augustana College, USA Prof. Drew Alton

Black Hills State University, USA Prof. Dan Durben, Prof. Kara Keeter, Prof. Michael Zehfus

Fermi National Accelerator Laboratory, USA Dr. Steve Brice, Dr. Aaron Chou, Dr. Jeter Hail, Dr. Hans Jostlein, Dr. Stephen Pordes, Dr. Andrew Sonnenschein

Princeton University, USA Jason Brodsky, Prof. Frank Calaprice, Huajie Cao, Alvaro Chavarria, Ernst de Haas, Prof. Cristiano Galbiati, Eng. Augusto Goretti, Eng. Andrea lanni, Tristen Hohman, Ben Loer, Pablo Mosteiro, Prof. Peter Meyers, Eng. David Montanari, Allan Nelson, Eng. Robert Parselis, Richard Saldanha, Eng. William Sands, Dr. Alex Wright, Jingke $X u$

Temple University, USA Prof. Jeff Martoff, Prof. Susan Jansen-Varnum, Christy Martin, John Tatarowicz

University of California at Los Angeles, USA Prof. Katsushi Arisaka, Prof. David Cline, Chi Wai Lam, Kevin Lung, Prof. Peter F. Smith, Artin Teymourian, Dr. Hanguo Wang

University of Houston, USA Prof. Ed Hungerford and Prof. Lawrence Pinsky

University of Massachusetts at Amherst, USA Prof. Laura Cadonati and Prof. Andrea Pocar

University of Notre Dame, USA Prof. Philippe Collon, Daniel Robertson, Christopher Schmitt

University of Virginia, USA Prof. Kevin Lehmann

\section{INTRODUCTION}

There is a wide range of astronomical evidence that the visible stars and gas in all galaxies, including our own, are immersed in a much larger cloud of non-luminous matter, typically an order of magnitude greater in total mass. The existence of this "dark matter" is consistent with evidence from large-scale galaxy surveys and microwave background measurements, indicating that the majority of matter in the universe is non-baryonic. The nature of this non-baryonic component is still totally unknown, and the resolution of the "dark matter puzzle" is of fundamental importance to cosmology, astrophysics, and elementary particle physics [1-3].

A leading candidate explanation, motivated by supersymmetry theory, is that dark matter is comprised of as yet undiscovered Weakly Interacting Massive Particles (WIMPs) formed in the early universe and subsequently gravitationally clustered in association with baryonic matter. WIMPs could in principle be detected in terrestrial experiments through their collisions with ordinary nuclei, giving observable low-energy $(<100 \mathrm{keV})$ nuclear recoils. The predicted low collision rates require ultra-low background detectors with large ( $0.1-10$ ton) target masses, located in deep underground sites to eliminate neutron background from cosmic ray muons.

Among a large number of developing detector technologies, liquid noble gas time projection chambers (TPCs), which detect scintillation light and ionization generated by recoiling nuclei, are particularly promising. The signal/background discrimination power, the attainable precision of determining 3-D event positions, and the effectiveness of chemical purification and cryogenic distillation methods for $\mathrm{Ar}$ and $\mathrm{Xe}$ have been demonstrated in published results from many members of the present collaboration. The Princeton group participates in the WARP collaboration and has contributed to the operation of a $3.2 \mathrm{~kg}$ Ar prototype reaching a sensitivity of $10^{-42} \mathrm{~cm}^{2}$ in a $96 \mathrm{~kg}$.day run [4]. This effort has been succeeded by a $140 \mathrm{~kg}$ Ar detector, WARP-140, which was commissioned in 2009 and is the largest noble liquid WIMP detector to date. The projected sensitivity of for WARP-140 in a 6-month run is $1 \times 10^{-44} \mathrm{~cm}^{2}$. The UCLA group participated in the ZEPLIN-II detector [5], which set a limit of $<6 \times 10^{-42} \mathrm{~cm}^{2}$ in 2006. The UCLA group is now participating in the XENON-100 experiment [6], currently operating at LNGS and expected to reach a cross section sensitivity of $\sim 10^{-45} \mathrm{~cm}^{2}$ in 7 months of running.

We propose to develop and operate a new liquid argon detector for WIMP detection, the first to employ argon with low levels of ${ }^{39} \mathrm{Ar}$, together with innovations in photon detection and background suppression. The new technology will be used for a low-background, high sensitivity $50 \mathrm{~kg}$ detector, the DARKSIDE-50, but it also makes possible large multi-ton detectors with high sensitivity for WIMP detection. The new detector will make use of the following new features: 
- Underground argon with low levels of ${ }^{39} \mathrm{Ar}$

- Low-background, high quantum efficiency QuPID photo detectors

- A compact high-efficiency neutron veto.

The use of DAr is required if ton-scale LAr TPCs are to be built. Ordinary atmospheric argon contains ${ }^{39} \mathrm{Ar}$, a $\beta$-emitter $(Q=565 \mathrm{keV}, \tau=388 \mathrm{yr})$ produced by cosmic rays, with the isotopic abundance ${ }^{39} \mathrm{Ar} / \mathrm{Ar}=8 \times 10^{-16}$, corresponding to a specific activity of $\sim 1 \mathrm{~Bq} / \mathrm{kg}[7,8]$. The most powerful nuclear recoil identification in $L A r$ uses scintillation pulse shape discrimination requiring clean measurements of individual pulses out to several microseconds duration (see below for details). Event pile-up due to the long drift time of electrons limits the size of unsegmented atmospheric argon detectors to about 1 ton. Satisfactory nuclear recoll discrimination in bigger detectors can only be achieved by lowering the specific activity of the argon by using ${ }^{39} \mathrm{Ar}$ depleted material.

Recently, the Princeton and Notre Dame DARKSTDE groups have demonstrated extraction of just such DAr gas from underground sources. (This work was done with ongoing NSF support.) This argon can now be produced in sufficient quantity and at low enough cost [9] to allow us to propose operating the DARKSIDE-50 detector with a DAr fill. The DARKSIDE-50 program will benefit from NSF grant PHY-0811186, DUSEL R\&D: Depleted Argon from Underground Sources, awarded to Princeton University in August 2009, which provided funds for the construction of an argon extraction and purification facility and for the collection of a batch of several tens of $\mathrm{kg}$ of depleted argon. The same grant provided funds for the construction of a UHV evaporator for the wavelength shifter in use to convert the $128 \mathrm{~nm}$ argon scintillation light to the visible range

All conventional photo detectors emit neutrons. The resulting background can limit the ultimate sensitivity of noble liquid TPCs since neutron-induced nuclear recoils are indistinguishable from WIMP interactions. Our UCLA collaborators have worked with Hamamatsu to invent and develop a new ultra-low radioactivity photo detector the Quartz Photon Intensifying Detector (QUPID). Factory prototype QUPID devices have been produced with radioactivity 100 times lower than the lowest activity PMTs available. The device does not require a resistive divider chain, removing another potentially important background source. These features allow QuPIDS to be placed in close contact with the active DAr, avoiding the need for an intervening thick, transparent neutron shield. Light collection and position resolution are dramatically improved and the energy threshold is significantly reduced.

A limiting background for all dark matter detectors is the production in their active volumes of nuclear recoils from the elastic scattering of background neutrons. An active veto with a high neutron detection efficiency allows a large fraction of these recoil backgrounds to be identified and removed. This not only reduces the direct background in the experiment, but the in situ background measurement provided by the active veto also makes the prediction of the residual, un-vetoed, neutron recoil background much more concrete.

We have submitted LOIs to LNGS, Sanford Laboratory, and SNOLab to deploy the DARKSIDE-50 detector. The experiment is conceived and designed to cope with background (from rock and cosmogenics) in any of the three locations. We have also discussed the possibility of performing initial tests in the KURF Laboratory. See the letters of support from the directors of LNGS, SNOLab, and KURF for an up-to-date status of our discussions. We anticipate a decision on the location of the experiment in the beginning of 2010 .

\section{OVERVIEW OF THE DEPLETED ARGON TPC DETECTOR}

In this experiment the active medium for detection of dark matter WIMPS is liquid argon, a cryogenic material with excellent scintillation and ionization properties. If WIMPs exist they are expected to collide with nuclei and produce a recoil atom with kinetic energies up to $100 \mathrm{keV}$. The recoil atom produces a short track of ionization and metastable atoms. After the initial ionizing event, a sequence of reactions occurs that involves recombination of electron-ion pairs and formation of excited diatomic molecules that decay with the emission of 128 -nm scintillation photons. There also remain free electrons that have not recombined.

The event is detected by observing both the scintillation photons and the free electrons. For background events resulting in a recoiling electron, such as beta or gamma events, the low density of electron-ion pairs results in less recombination and therefore less scintillation and more free electrons, as compared to a nuclear recoil track of high ionization density [10-12]. The ratio of ionization to scintillation thus allows separation of background events due to electron recols from those due to nuclear recoils.

The difference in ionization density between nuclear and electron recoils also produces a significant difference in the time profile of the scintillation light $[10,13]$. Scintillation photons are emitted from two nearly degenerate states, a long-lived ( $\sim 6 \mu \mathrm{s}$ ) triplet state, and a short-lived ( $\sim 6 \mathrm{~ns}$ ) singlet state. The long-lived state is found 


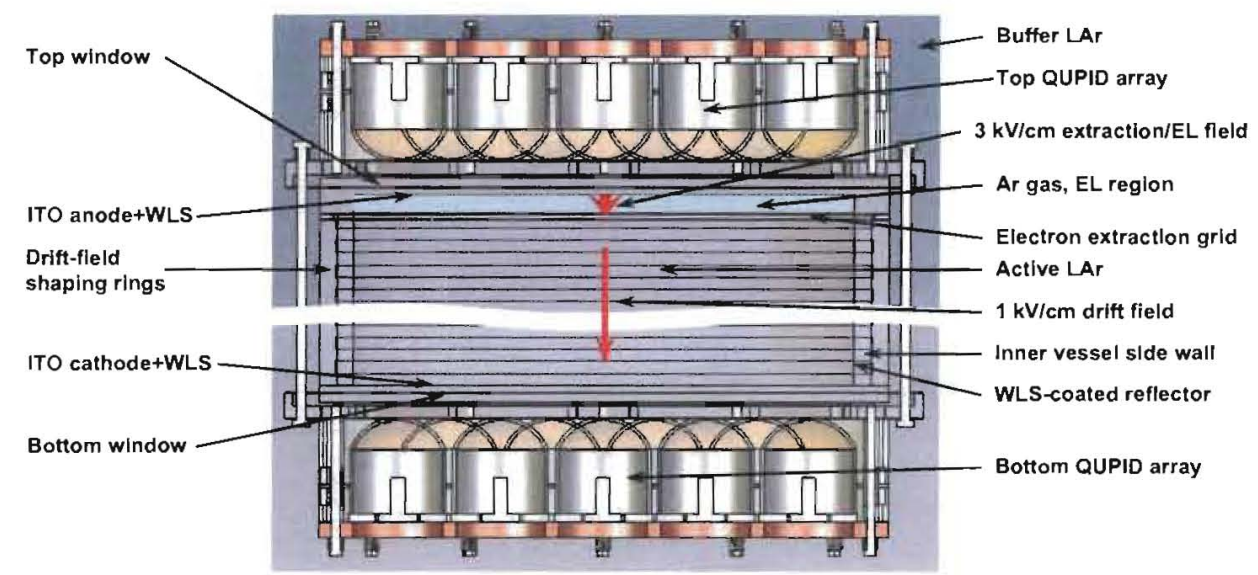

FIG. 1: The two-phase TPC for liquid argon. For clarity in viewing details in the upper and lower sections, part of the middle of the detector has been removed.

to be quenched in tracks with high ionization density. Thus, electron recoils have longer scintillation duration compared to nuclear recoils, providing a very powerful "pulse shape discrimination" (PSD) between electron backgrounds and nuclear-recoil signal [14]. The combination of discrimination by the ionization to scintillation ratio with the pulse shape discrimination provides powerful background rejection that is unique to argon. To exploit these powerful background suppression characteristics requires a two-phase "two-phase time projection chamber" (TPC) made of low radioactivity components and with high light- and ionization-collection efficiency.

Figure 1 shows the proposed inner detector. We provide a short overview of its parts and functionality here; more details can be found in Sec. IX. The inner detector contains the active liquid argon volume. Arrays of photo detectors, immersed in the buffer liquid argon surrounding the detector, view the active volume from the top and bottom. Each array consists of nineteen 3-inch diameter QUPID photo detectors (see Sec. IV). A tetra-phenylbutadiene (TPB) wavelength shifter (WLS) is used to shift the $128 \mathrm{~nm}$ UV scintillation photons to the visible for detection

A TPC configuration is employed to detect the ionization electrons. The electrons drift upward in the liquid under the influence of a uniform $\sim 1 \mathrm{kV} / \mathrm{cm}$ electric field produced by a "field cage" consisting of a cathode plane, field-shaping rings, and an extraction grid. An electric field of $\sim 3 \mathrm{kV} / \mathrm{cm}$ extracts the electrons into the gas phase, where they produce secondary scintillation photons by a process called "electroluminescence" $(E L)[12,15]$. The secondary photons (S2) are detected by the photo detector array as a delayed coincidence relative to the primary scintillation (S1). The TPC also provides complete 3D-position information for the event. Diffusion during the long drift is negligible in the dense noble liquids [11] - the drift time gives the vertical position to sub-millimeter precision, and the pattern of photons on the top photo detector array gives the horizontal position to $<1 \mathrm{~cm}$.

Two-phase DAr TPCs with low background photosensors should allow construction of affordable multi-ton detectors operating with zero accepted background in multi-year exposures. The suppression is achieved by fully exploiting the rich information content of the combined ionization and scintillation signals available from the TPCs. We list here established performance of existing detectors; estimates of the performance of the proposed DARKSIDE-50 detector are given in Sec. IX.

1. Pulse Shape Discrimination. This technique has been proved to reject $\beta / \gamma$ background by a factor of up to $6 \times 10^{8}[4,16]$. The PSD background rejection depends critically on the number of photons detected [14], which depends in turn on the photosensor coverage and the photosensor quantum efficiency. The larger the photo-electron yield, the lower the threshold can be while retaining adequate discrimination.

2. Scintillation to Ionization Ratio. The $S 2 / S 1$ ratio provides a factor of $\sim 200-1000$ separation between nuclear recoils and other event types [4]. This and the self-shielding provided by the dense target are the only electron rejection methods available to liquid xenon TPCs.

3. Position Reconstruction and Fiducialization. Precise event localization, which is not available in singlephase detectors, is extremely important. In large detectors it allows rejection of most neutron interactions, which will usually show multiple interaction sites. Localization also allows rejection of backgrounds from radioactivity on the surface of the fieid cage that are primarily associated with radon daughters. 

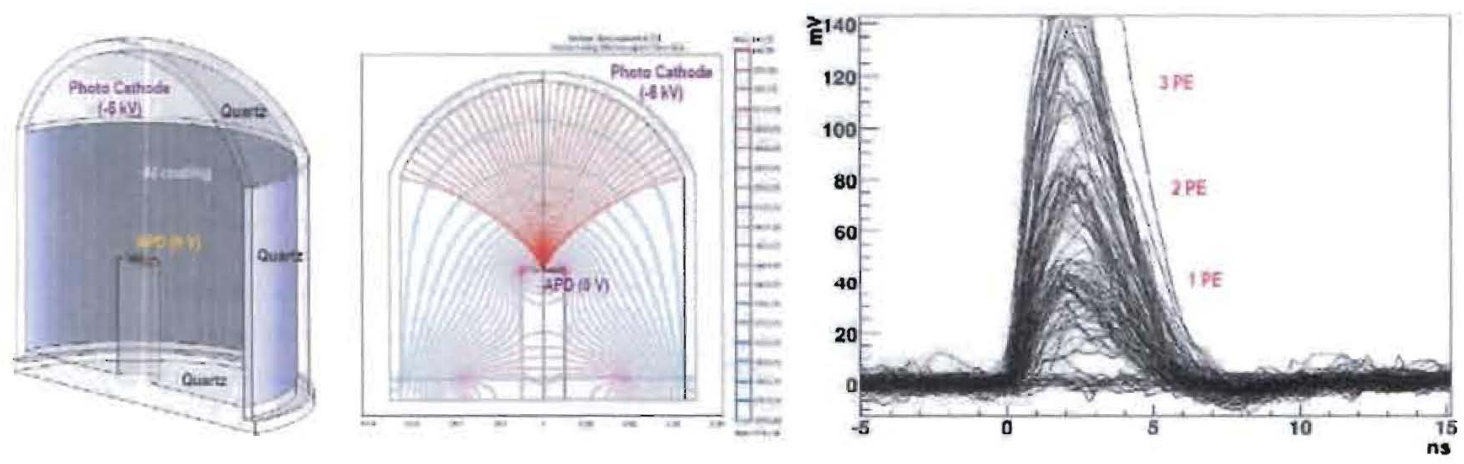

FIG. 2: a) Structure of the QUPID. b) Electron optics of the QUPID. c) Digitized QuPID waveforms: note the separated bands corresponding to detection of 1,2 , and 3 photoelectrons.

\section{QUPIDS}

The DARKSIDE-50 detector will be instrumented with the revolutionary new QUPID photo detectors, which provide extraordinarily low background and excellent quantum efficiency and time resolution. QuPID characteristics determined from tests of actual prototypes are summarized in Table I.

The photo detectors required to instrument noble liquid TPCs are usually among the dominant source of $\gamma$-rays and neutrons inside the detector. The success of multi-ton noble liquid TPCs thus depends on the development of ultra-low-radioactivity, high-efficiency photon detectors. To address this challenge, the UCLA group collaborated with Hamamatsu to invent and produce prototypes of an innovative photon detector, the Quartz Photon Intensifying Detector (QUPID).

As shown in Figure 2a, the QUPID is a hybrid PMT. Photoelectrons emitted from the 3" diameter hemispherical photocathode are accelerated to $6 \mathrm{kV}$ and focused onto an Avalanche PhotoDiode (APD). The total charge gain resulting from the acceleration plus the APD avalanche is $2 \times 10^{5}[17,18]$. The QUPID envelope is fabricated from low-radioactivity synthetic fused silica. These devices can drive several meters of cable without pre-amplification, while preserving very sharp timing characteristics (transit time spread=250 psec, rise time $=1.3 \mathrm{nsec}$, fall time $=2.6 \mathrm{nsec}$, and pulse width $F W H M=3.1 \mathrm{nsec}$ ). A simple readout scheme can therefore be used, with a linear amplification stage followed by direct digitization of the waveform. Moreover, thanks to the high noiseless gain from the electron acceleration, the signal-to-noise ratio is high enough to reveal clear peaks corresponding to not only single- but also of two- and three-photoelectron events (see Figure 2c).

The first four prototype QUPIDs were screened for radioactivity in a 1-month run in the Germanium-based screening facility dedicated to XENON-100 at LNGS. The radioactivity of the QUPID was too low to be detected above the intrinsic background of the detector, resulting in a $95 \%$ C.L. limit of $<3.5 \mathrm{mBq}$ (see Table I). This upper limit can be compared to the activity in PMTs used in current dark matter TPCs: $58 \mathrm{mBq}$ per 3" Metal Bulb PMT (MB-PMT) Hamamatsu R11065, 15 mBq total activity per 2" PMT Hamamatsu R8778, or $\sim 1 \mathrm{mBq}$ total activity per 1" PMT Hamamatsu R8520-06-AL, used in XENON-100. The corresponding neutron emission rate from the QUPID (as calculated with the SOURCES package [19]) is less than $10^{-3} \mathrm{n} /\left(\mathrm{yr} \cdot \mathrm{cm}^{2}\right)$, a limit more than ten times better than the rate for the 1" PMT Hamamatsu R8520.

The photosensors must operate at cryogenic temperatures where standard bialkali photocathodes can have extremely low saturation currents. Hamamatsu has developed a breakthrough "Bialkali-LT" (Low Temperature) photocathode, which operates down to LAr temperatures with high Quantum Efficiency (QE). Figure 3a illustrates the difference in saturation current performance at liquid argon and liquid xenon temperature between the Bialkali-LT and a traditional bialkali photocathode. The Bialkali-LT photocathode achieves this high saturation

\begin{tabular}{|c|c|c|c|c|c|c|c|c|}
\hline & \multicolumn{5}{|c|}{ Radioactivity } & \multicolumn{3}{|c|}{ Photocathode } \\
\hline & $\begin{array}{c}238 \mathrm{U} \\
{[\mathrm{mBq}]}\end{array}$ & $\begin{array}{l}{ }^{2.32} \mathrm{Th} \\
{[\mathrm{mBq}]}\end{array}$ & $\begin{array}{c}{ }^{40} \mathrm{~K} \\
{[\mathrm{mBq}]}\end{array}$ & $\begin{array}{l}{ }^{60} \mathrm{Co} \\
{[\mathrm{mBq}]}\end{array}$ & $\begin{array}{c}\text { Neutrons } \\
{[n / \mathrm{yr}]}\end{array}$ & Type & $\begin{array}{c}\text { T Range } \\
{[\mathrm{K}]}\end{array}$ & $\begin{array}{l}\text { QE } \\
{[\%]}\end{array}$ \\
\hline 3" QUPID & $<0.49$ & $\leq 0.40$ & $<2.40$ & $<0.21$ & $<0.05$ & Bialkali-LT & $70-330$ & 37 \\
\hline
\end{tabular}

TABLE I: Characteristics and background of the 3" QUPIDs. All limits are quoted at 95\% C.L. and refer to radioactive contamination or neutron rates for a single photo detector. 

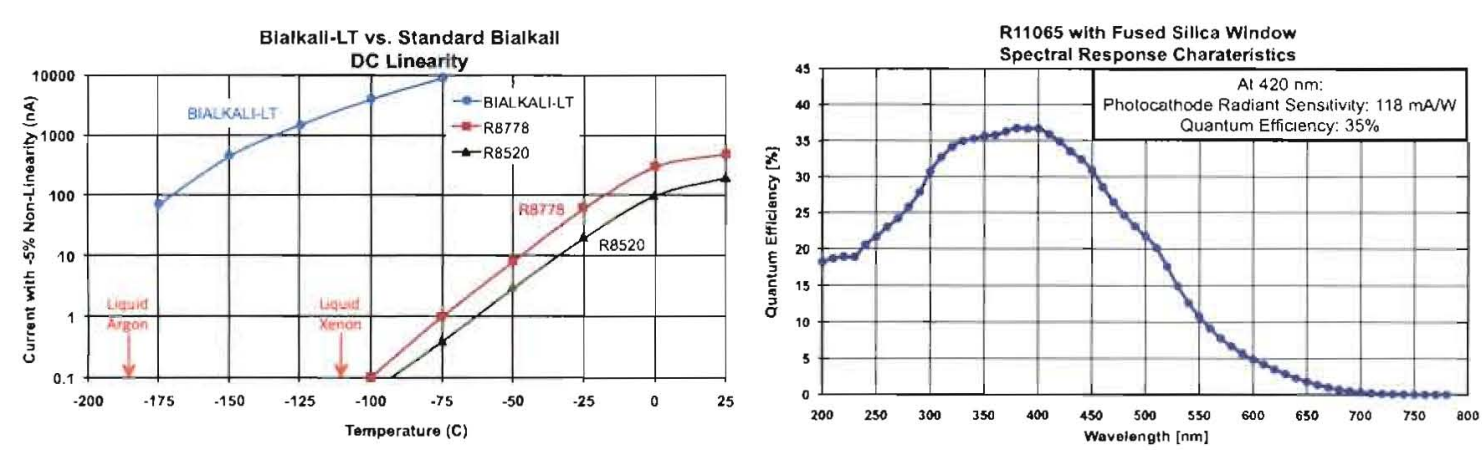

FIG. 3: a) DC linearity for the Bialkali-LT photocathode versus temperature, compared with the same quantity for the PMTs R8778 and R8520, equipped with traditional bialkali photocathodes. b) Quantum efficiency of the Bialkali-LT photocathode on the fused silica window of the 3" MB-PMT R11065.

current without the large QE penalty (QE $\leq 20 \%$ ) incurred by the standard solution for low temperature operation (depositing a thin platinum conducting layer over the photocathode). Hamamatsu, under contract from Princeton University, optimized deposition of the Bialkali-LT photocathode on $3^{\prime \prime}$ substrates in the production of a limited set of R11065 metal-bulb PMTs with QE up to $35 \%$ at $420 \mathrm{~nm}$ (see Figure 3b).

The DARKSIDE-50 program will strongly benefit from R\&D performed for MAX, funded under the NSF DUSEL S4 program. Funding for MAX includes a subcontract to Hamamatsu ( $\$ 300 \mathrm{k}$ over three years) for studies on mass production of QUPIDs, optimization of their quantum and collection efficiency, and optimization of their operation at liquid argon temperature. Under this program, Hamamatsu will also perform a study of feasibility of producing 6" and 8" QUPIDS (see their letter of support).

In DARKSIDE-50, 38 3" QuPIDs will be used, 19 each on the top and bottom of the TPC.

\section{COLLECTION AND PURIFICATION OF DEPLETED UNDERGROUND ARGON}

Centrifugation or differential thermal diffusion are established methods for ${ }^{39} \mathrm{Ar} /{ }^{40} \mathrm{Ar}$ isotopic separation. However, with very high costs $(\$ 40 \mathrm{k} / \mathrm{kg})$ and a global production capacity of a few $\mathrm{kg} / \mathrm{month}$, these options are not practical for large detectors. Since ${ }^{39} \mathrm{Ar}$ is produced in the atmosphere by cosmic ray interactions on ${ }^{40} \mathrm{Ar}$, such as ${ }^{40} \operatorname{Ar}(n, 2 n){ }^{39} \mathrm{Ar}$, one might expect that a source of underground argon which has been protected from cosmic rays for many ${ }^{39} \mathrm{Ar}$ half-lives would have a very low ${ }^{39} \mathrm{Ar}$ content. The Princeton group, in a 2 -year NSF-sponsored R\&D program $[9,20,21]$, has identified two such underground sources of argon-containing gas capable of producing in excess of 30 tons of argon per year, at an anticipated cost of $\sim \$ 0.5-1.0 \mathrm{k} / \mathrm{kg}$ for a 5 ton batch.

The two underground argon sources, the National Helium Reserve (Amarillo, TX) and the Doe Canyon $\mathrm{CO}_{2}$ Formation (Cortez, CO), were sampled during 2008, using a two-stage VPSA system designed by Koch Modular Process Systems (KPMS) and built by Princeton University. The argon samples were assayed by low level counting, obtaining respective upper limits of $5 \%$ and $4 \%$ for the ${ }^{39} \mathrm{Ar}$ isotope content relative to that of atmospheric argon. While the present upper limits on the ${ }^{39} \mathrm{Ar} / \mathrm{Ar}$ ratio more than satisfy the requirements of the present experiment, we believe that the ultimate ${ }^{39} \mathrm{Ar} / \mathrm{Ar}$ ratio at both sources could be much lower than this upper limit. Members of the collaboration are building a low-background ${ }^{39} \mathrm{Ar}$ counter with a $1 \mathrm{~kg}$ depleted argon target mass that will improve the sensitivity to $0.1 \%$ of the atmospheric activity [20-22]

Owners of the gas streams from both sources (Linde USA, drawing crude helium gas from the National Helium Reserve, at its Global Helium plant in Otis, KS; the Kinder Morgan Corporation, owning mining rights at Doe Canyon) support the effort. The preferred option for the depleted argon production is the Doe Canyon $\mathrm{CO}_{2}$ formation. The Office of Research and Project Administration of Princeton University and the Office of General Counsel of Kinder Morgan have executed a "Facility Access Agreement" that allows Princeton researchers to perform the extraction on the Kinder Morgan premises in Cortez, CO. With seed funding provided by Princeton University, we have installed and commissioned a plant capable of producing $0.5 \mathrm{~kg} /$ day of depleted argon at the Kinder Morgan facility, shown in Fig. 4. The DARKSIDE-50 program will leverage NSF grant PHY0811186, DUSEL R\&D: Depleted Argon from Underground Sources, which in August 2009 provided $\$ 1.6 \mathrm{M}$ to Princeton University for the expansion of the argon extraction and purification facility and for the collection of 


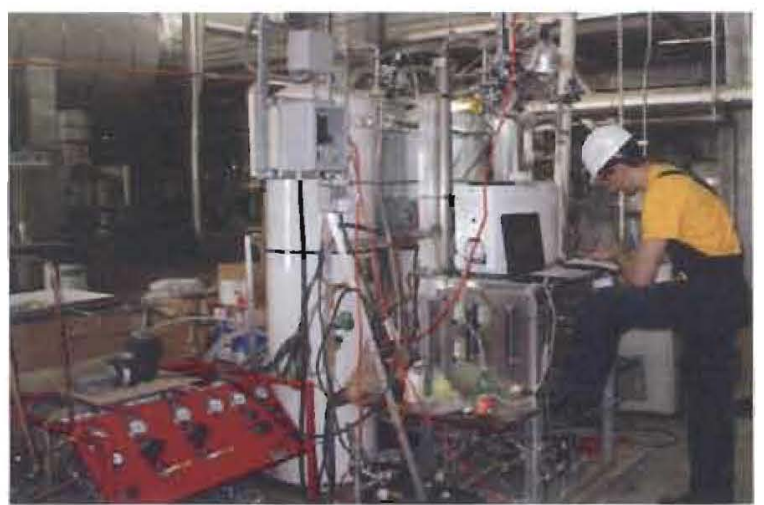

FIG. 4: Two-stage Vacuum and Pressure Swing Adsorption plant developed at Princeton. The plant was operated on the Cortez $\mathrm{CO}_{2}$ stream for one week in May 2009, producing at the rate of $0.5 \mathrm{~kg}$ per day a total of $\sim 3 \mathrm{~kg}$ of argon depleted by a factor 25 or more (relative to atmospheric argon). Princeton engineer D. Montanari is seen at the controls.

a batch of several tens of $\mathrm{kg}$ of depleted argon. A first upgrade, supported by the new grant and designed to bring the production rate to $\sim 2 \mathrm{~kg}$ /day and to test the recovery of the $\mathrm{CO}_{2}$ separated from the argon, is already in progress and is scheduled for completion by December 2009. A second upgrade, scheduled for 2010, may bring the production rate above $10 \mathrm{~kg} /$ day. The DARKSIDE- 50 program will also leverage the MAX effort, whose funding supports a subcontract to Linde ( $\$ 150 \mathrm{k}$ over three years) for a simulation of the argon extraction plant, in view of its upgrade towards a DUSEL-scale facility. Results of the Linde simulations will be instrumental in guiding the argon extraction and collection at all stages

DAr is extracted from an underground natural gas stream in which the argon starts out as only a minor constituent ( $\sim 0.01-0.1 \%$ in volume) and requires several stages of purification before it is suitable for detectors. Depleted argon (DAr) is first refined at the well head into a crude mixture with typical argon concentration $\sim$ $10 \%$ by volume, with the remainder largely $\mathrm{N}_{2}, \mathrm{CH}_{4}$, and $\mathrm{He}$.

The most effective method to remove these impurities is cryogenic distillation. Under a contract from Princeton University, Koch Modular Process Systems, LLC. performed a preliminary study which showed that a single $60-$ stage cryogenic distillation unit is capable of removing $\mathrm{N}_{2}, \mathrm{CH}_{4}$, and $\mathrm{He}$ from $\mathrm{Ar}$, which maintaining $>95 \%$ Ar recovery. Support from two Linde engineers (Frank Fitch and Stevan Jovanovic) allowed completion of the study, resulting in the design of a very compact unit. Supported by funds provided by NSF grant PHY0811186, construction of the cryogenic distillation column has already started and is scheduled for completion by March 2010. The column will process the "crude" depleted argon extracted at the Doe Canyon $\mathrm{CO}_{2}$ facility and produce "6 nines" (99.9999\%) purity argon.

\section{A COMPACT, HIGH-EFFICIENCY ACTIVE NEUTRON VETO}

Primary sources of background external to the cryostat are: 1) muons, producing $\gamma$-rays and high energy neutrons either in the rocks surrounding the lab or in the shielding material; 2) airborne contaminants such as ${ }^{222} \mathrm{Rn}$ and its daughters and ${ }^{85} \mathrm{Kr}$; 3) $\mathrm{U}, \mathrm{Th}$, and $\mathrm{K}$ in the rocks, producing $\gamma$-rays and neutrons from fission and $(\alpha, n)$ reactions; 4$) \mathrm{U}, \mathrm{Th}$, and $\mathrm{K}$ in the shielding material and in the external parts of the detector, producing $\gamma$-rays and neutrons.

An experiment attempting to directly detect dark matter through WIMP-nucleon scattering must have carefully designed shielding to reduce the background from neutrons and gamma-rays. For DAr detectors, the excellent gamma-discrimination described earlier makes the gamma background less troublesome than the neutron background. The ultimate background for any dark matter detector are neutrons which can elastically scatter to produce single recoil nuclei in the detector. These neutron-induced recoils are indistinguishable from WIMP interactions.

Passive shielding can reduce the level of neutron-induced nuclear recoils to levels sufficient for WIMP searches to be carried out. However, it is challenging for an experiment with a passive shield to conclusively demonstrate the background level that has been achieved, which in turn makes the interpretation of a few observed recoil events as a WIMP signal problematic. A superior method of neutron suppression is the use of an active neutron veto 


\begin{tabular}{lclc}
\hline \hline Component & Characteristics & Component & Characteristics \\
\hline Passive Shield Inner Length & $256 \mathrm{~cm}$ & Passive Shield Outer Length & $326 \mathrm{~cm}$ \\
Passive Shield Inner Width & $256 \mathrm{~cm}$ & Passive Shield Outer Width & $326 \mathrm{~cm}$ \\
Passive Shield Inner Height & $256 \mathrm{~cm}$ & Passive Shield Outer Height & $361 \mathrm{~cm}$ \\
Inner Steel Layer Thickness & $7.5 \mathrm{~cm}$ & Inner Steel Layer Mass & $26 \mathrm{~T}$ \\
Lead Layer Thickness & $20 \mathrm{~cm}$ & Lead Layer Mass & $120 \mathrm{~T}$ \\
Outer Steel Layer Thickness & $7.5 \mathrm{~cm}$ & Outer Steel Layer Mass & $38 \mathrm{~T}$ \\
Scintillator Tank Diameter & $256 \mathrm{~cm}$ & Scintillator Tank Height & $291 \mathrm{~cm}$ \\
Scintillator Mass & $12 \mathrm{~T}$ & Scintillator Boron Loading & $10 \%(\mathrm{w} / \mathrm{w})$ \\
Number of 8" Veto PMTs & 50 & & \\
\hline \hline
\end{tabular}

TABLE II: Shield dimensions and characteristics

(the "neutron veto") in which the neutrons are detected with very high efficiency and corresponding recoil events induced by neutrons in the argon are thus identified and rejected. Apart from the direct background suppression, an active veto also provides an in situ measurement of the true neutron background in the experiment, which makes the prediction of the number of neutron-induced recoils that are not vetoed much more concrete. This precise understanding of the neutron background would significantly increase the credibility of any claim of the detection of a dark matter signal, and will also allow direct extrapolation of the background levels achieved in DARKSIDE- 50 to larger detectors.

Typical active neutron vetos surrounding xenon detectors have an efficiency of $\sim 60 \%$ [5]. In the case of WARP-140, the efficiency of the veto is $>98 \%$, but at the expense of an extremely massive active veto, which increases the the inventory of the liquefied noble gas to 150 times the mass of the dark matter target: 25 tons of Liquid Argon ( 9 tons in the active veto and 16 tons of inactive buffer), to be compared to a target of $140 \mathrm{~kg}$.

Here we propose the construction of a compact, liquid scintillator-based, high efficiency neutron veto. Neutrons most likely to produce events mimicking WIMP-induced recoils enter the active target with energies in the $1-5 \mathrm{MeV}$ range. At these energies, neutrons are efficiently thermalized (within a few tens of $\mathrm{cm}$ ) by highly hydrogenated media, such as paraffin, HDPE, or liquid scintillator. Many competing proposals rely on Gd-loaded scintillator to enhance the energy of $\gamma$-rays from neutron capture. This enhancement, however, is achieved at the expense of a serious loss of overall efficiency, as energetic $\gamma$-rays from capture on Gd or even on protons tend to travel a distance significantly further than neutrons in the $1-5 \mathrm{MeV}$ range. Key to our proposal is the idea to abandon the use of $(n, \gamma)$ capture agents and rely instead on capture in nuclides that produce short-ranged radiation in form of charged particles.

One such nuclide is ${ }^{10} \mathrm{~B}$ : the dominant cross section for neutron interaction is through the $(n, \alpha)$ channel, producing $\alpha$ 's of energy $1.47 \mathrm{MeV}$ or larger. Studies on the loading of boron into organic liquid scintillator were performed in the 80's, when trimethylborate was the first candidate target for BOREX [23], the progenitor of the solar neutrino experiment BOREXINO [24]. The same compound is now finding application in neutron detectors [25]. We note that since the isotopic abundance of ${ }^{10} \mathrm{~B}$ is $20 \%$, and the cross section for $(n, \alpha)$ on ${ }^{10} \mathrm{~B}$ is thousands of times larger than the capture cross section on protons and on ${ }^{11} \mathrm{~B}$, no isotopic enrichment is required.

We have designed our neutron veto as a $1-\mathrm{m}$ thick liquid scintillator volume, loaded to $10 \%$ by mass with natural boron, surrounding the detector cryostat. We have performed Monte Carlo studies of the veto efficiency in this geometry, and obtain an efficiency of $>99 \%$ in vetoing background events in the argon from $1-10 \mathrm{MeV}$ neutrons. This is in line with estimates [26-28] of the neutron detection efficiency of the commercially available boron-loaded scintillator BC523A [29]. We note that the same scintillator is also expected to be an excellent detector for faster neutrons, exploiting the elastic scattering of neutrons on protons [28]. In fact, Monte Carlo studies suggest that the scintillator veto will have $>95 \%$ efficiency in vetoing background events induced by energetic cosmogenic neutrons (we took into account in our simulations the energy-dependent quenching of light from fast protons as per Ref. [30]).

Neutron captures on ${ }^{10} \mathrm{~B}$ deposit in the scintillator at least $65 \mathrm{keV}_{\mathrm{ee}}$ [31] from the recoils of the resulting $\alpha$ particles and ${ }^{7} \mathrm{Li}$ daughters (this corresponds to an overall quenching of $\sim 40$ for the combined recoil products). We plan to equip the veto tank with 50 8" PMTs, corresponding to a $5.5 \%$ photocathode coverage, and to cover all internal surfaces of the tank with diffuse reflectors with reflectivity $>95 \%$ to recycle the light not hitting the PMTs directly. For a scintillator light yield of 5,000 produced photons per $\mathrm{MeV}_{\text {ee }}$ and a QE of $\sim 25 \%$ for the PMTs we expect to obtain $\sim 210$ p.e. $/ \mathrm{MeV}_{\text {ee }}$. Therefore, $65 \mathrm{keV}_{\text {ee }}$ recoils would produce $\sim 15$ p.e., which is easily detectable. 
Active vetos can function effectively with a relatively high event rate, as high as a few hundred events per second. This means that the liquid scintillator veto would require only a relatively small amount of external shielding. As little as $15 \mathrm{~cm}$ of steel and $20 \mathrm{~cm}$ of lead would be sufficient to reduce the rate of rock $\beta / \gamma$ backgrounds to a level tolerable to the veto. Monte Carlo simulations suggest that the veto rate in this geometry would be $\approx 300 \mathrm{~Hz}$. The steel and lead outer shielding is referred to as the "passive shield". Minimal precautions in the design of the passive shield would allow flushing of the volume between the passive shield and the tank with ultrampure nitrogen gas to reduce background from ${ }^{222} \mathrm{Rn}$ and ${ }^{85} \mathrm{Kr}$.

Construction of the neutron veto is a very significant project, tantamount to the construction of a separate detector. In order to meet the desired schedule, which calls for the start of data taking with the first depleted argon detector in the Spring of 2011 (see Section IX), first operation of the DAr TPC will happen within the bare passive shield, with the TPC surrounded by an inner layer $(5 \mathrm{~cm}$ thick) of copper and an outer layer (40-cm thick) of HDPE as external neutron moderator. This will permit neutron-free data taking for a few months. Upon completion of the TPC and start of the data taking in Spring 2011, part of the collaboration will engage in the construction of the active veto. We anticipate at that time the additional involvement of the two Princeton engineers stationed at LNGS and currently active in the purification of the Borexino target; a specific request will be formulated at the time of renewal of the Borexino grant, in Fall 2010. The demonstration of the effectiveness of the neutron veto is a crucial part of this proposal, which aims to deploy background-reducing technologies sufficient to allow a robust discovery of dark matter at the sensitivities to be probed by multi-ton detectors. If successful, this will become the baseline for the design of MAX at DUSEL.

Depending on the depth at which the experiment is ultimately operated further suppression of the background from neutrons in the 10-500 MeV range produced by cosmic rays interacting in the passive shield may be required to reach zero-background conditions. For operations at LNGS or at Sanford Lab, we plan the installation of an active muon veto (the "muon veto") surrounding five of the six sides of the passive shield, to veto muons that produce cascades in the passive shield without producing signals in the active liquid scintillator veto. The CDMS collaboration has demonstrated that a $2^{\prime \prime}$-thick plastic scintillator is sufficient to achieve good separation between cosmic rays signals and natural radioactivity, and that a veto based on this technology can achieve a $99.9 \%$ efficiency [32], significantly larger than the $99 \%$ efficiency required for the DARKSIDE-50 detector at LNGS (the shallowest of the sites under consideration). We include in our budget the capital cots of a plastic scintillatorbased muon veto with a $99 \%$ efficiency or larger. We will also explore other, potentially lower cost, methods of muon veto construction. We also note that at most a rudimentary muon veto will be required for operation of the detector at SNOLab, given the up-front reduction of the cosmic ray flux due to the greater depth.

\section{MITIGATION OF SURFACE BACKGROUND AND CONTAMINATION}

Mitigation of surface backgrounds is crucial for the success of the experiment. Radioactive daughters of ${ }^{222} \mathrm{Rn}$ plate out on surfaces and are the major contributors to surface $\alpha$ activity. Cross sections for ( $\alpha, n$ ) reactions result in one in every $10^{6}-10^{7} \alpha$-decay producing a low-energy neutron [33]. $\alpha$-decays on the inner surface of the detector are particularly dangerous for another reason - about half the time, the a goes deeper into the surface, and the daughter nucleus recolls into the active volume, mimicking a WIMP recoil. All detector surfaces will therefore be pre-cleaned to minimize the radon daughter surface activity and also to remove particulates (another important source of $\alpha$ and $\beta / \gamma$ activity) and other surface contamination. Recolls resulting from the remaining surface activity will be strongly suppressed by eliminating their $\$ 2$ signals by preventing their ionization from entering the gas phase. This could be accomplished by adding a narrow charge collection ring to the electron extraction grid near the edge of the vessel.

Surface contamination can be effectively mitigated by locating the last steps of construction and assembly in a ${ }^{222} \mathrm{Rn}$ suppressed clean-room. The sole ${ }^{222} \mathrm{Rn}$ suppressed clean-room ( $\mathrm{IB} \mathrm{Bq} / \mathrm{m}^{3}$ of air) in the world exists at Princeton University, where it was built for the construction of the Borexino nylon vessels. Background on surfaces of detectors assembled in this room can be contained to $<10 \alpha \mathrm{s} /\left(\mathrm{m}^{2} \cdot \mathrm{d}\right)$ (see Refs. [34, 35]; the same result was achieved for the SNO NCD detectors [36]). We plan to pre-assemble the detector as much as possible in this special facility. This will reduce the requirements for quality clean space at the experimenta! site. 
VIII. CRYOGENIC SYSTEMS

We plan to operate the detector inside a triple-jacketed ultra pure titanium cryostat [37]. The space between the inner two jackets serves as the vacuum insulation. The space between the outer two jackets is filled with a 1 "-thick cellular polystyrene with a very low thermal heat conductance $(\sim 0.015 \mathrm{~W} / \mathrm{m} / \mathrm{K})$. In case of the failure of either of the two inner jackets, the presence of thermal insulator would prevent a rapid phase transition of DAr by reducing the boil-off rate to $\sim 5$ std liters/sec of DAr gas. We plan to run all cables through a transfer pipe connecting to a flange sited outside the shield; dog legs on the pipe minimize the penetration of external $\gamma$-rays and neutrons. All ceramic feedthroughs for signals and power (with the exception of low-radioactivity feedthroughs for HHV, made of titanium and HDPE) are located on the flange outside the shield to prevent unwanted background. A liquid-nitrogen cold head, buit entirely of titanium and copper for background mitigation, delivers a cooling power greater than $100 \mathrm{~W}$ at $86 \mathrm{~K} . \mathrm{LN}_{2}$ is fed to the cold head through titanium vacuum insulated pipes, is vaporized and heated inside the cold head, then transferred outside the shield where it is cooled and re-liquefied by a Gifford-McMahon cryocooler in an independent reservoir. The design of the internal TPC allows to use a very large fraction of the cooling power to purify and recirculate the DAr in the active volume.

The DARKSIDE-50 detector will be equipped with independent zero boil-off recovery and storage systems, similar to that devised for the MEG experiment [38] and capable of recovering and storing the total inventory of the noble target either in gas phase in high-pressure tanks or in liquid phase in a dewar equipped with redundant active cooling (a $\mathrm{LN}_{2}$ reservoir). The system will be used when emptying the detector. It will allow the rapid transfer of the noble target to the recovery system in case of problems with the structural integrity of the detector cryostat. The system will also allow recovery of the DAr target in case of problems or required maintenance of the cooling loop.

\section{DEPLETED ARGON TPC}

A conceptual drawing of the proposed DARKSIDE-50 detector is shown in Figs, $5 \mathrm{a}$ and $5 \mathrm{~b}$. This design is based on concepts that have been developed over many years and have been demonstrated by the successful runs of the WARP, XENON, and ZEPLIN chambers and in large single phase TPCS for neutrino detection $[4-6,39,40]$.

The active volume contains $50 \mathrm{~kg}$ ( $33 \mathrm{~kg}$ fiducial) of DAr and is fitted with field shaping structures (drift cathode, field cage, extraction grid). These structures drift and extract charge from the liquid into the gas and form a delayed scintillation signal $(S 2)$ by electroluminescence.

Nineteen QupIDS will be used on the top and on the bottom of the detector, separated from the active DAr by a thin acrylic or fused silica window. The windows are coated with Indium Tin Oxide, ITO, a transparent conductor that allows the windows to serve as the anode (top) and the cathode (bottom) of the TPC. Since neither acrylic nor quartz are transparent to the $128 \mathrm{~nm}$ argon scintillation light, the light must be shifted into the visible range by a TetraPhenylButadiene (TPB, peak emission at $420 \mathrm{~nm}$ ) layer lining the entire active volume. $A$ thin layer of the liquid argon between the photocathode face and the windows acts as an optical coupling. The cylindrical side wall of the active region TPC will also be acrylic or fused silica, with a reflecting liner coated on its inner surface with TPB.

Demountable seals operating at liquid argon temperatures must be provided for the windows of the transparent

\begin{tabular}{lclc}
\hline Component & Characteristics Component & Characteristics \\
\hline Active Liquid Volume Height & $36.0 \mathrm{~cm}$ & $3^{\prime}$ Qupids, Top & 19 \\
Gas Height & $1.0 \mathrm{~cm}$ & 3 QupIDs, Bottom & 19 \\
Active DAr Mass & $50 \mathrm{~kg}$ & Buffer DAr Mass & $88 \mathrm{~kg}$ \\
Drift Electric Field & $1.0 \mathrm{kV} / \mathrm{cm}$ & Extraction Field & $3.0 \mathrm{kV} / \mathrm{cm}$ \\
Active Volume Diameter & $36.5 \mathrm{~cm}$ & PTFE Reflectors Mass & $7.3 \mathrm{~kg}$ \\
Acrylic Tube and Field Cage, ID & $36.5 \mathrm{~cm}$ & Anode/Cathode Acrylic Flange Thickness & $2.0 \mathrm{~cm}$ \\
Acrylic Tube and Field Cage, OD & $42.5 \mathrm{~cm}$ & Acrylic Mass & $18 \mathrm{~kg}$ \\
Ti Cryostat, ID & $49.2 \mathrm{~cm}$ & Ti Cryostat, Height & $115 \mathrm{~cm}$ \\
Ti Cryostat, OD & $55.5 \mathrm{~cm}$ & Ti Cryostat, Mass & $73.9 \mathrm{~kg}$ \\
Copper Qupios Holder Mass & $11.3 \mathrm{~kg}$ & & \\
\hline
\end{tabular}

TABLE III: Depleted argon TPC: detector dimensions and other parameters. 


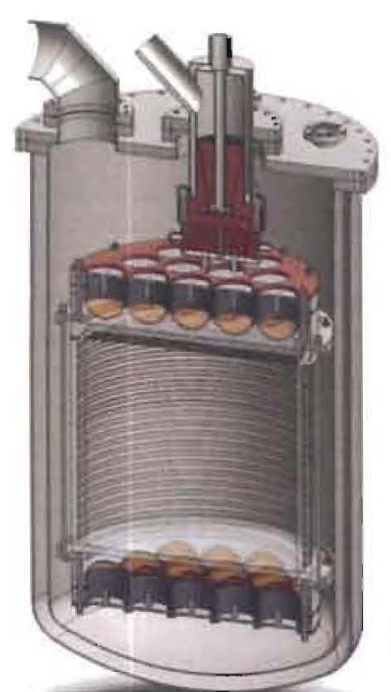

FIG. 5: (a) The DARKSIDE-50 internal detector. (b) The DARKSIDE-50 detector within the active liquid scintillator neutron veto and the passive shield.

vessel. Spring-energized O-ring seals have been found to produce acceptable results on small diameter acrylic joints, and it is anticipated that this will work for the larger diameter seals. A "diving bell" arrangement below the anode window maintains the level between the gas and liquid phases. The baseline design uses a conventional field cage outside of the transparent vessel. The drift cathode must be supplied with a potential of $\sim 50 \mathrm{kV}$. The required "High-High Voltage" (HHV) feedthrough from air into liquid argon will be based on the successful $150 \mathrm{kV}$ feedthrough for Icarus T-600, designed by our UCLA colleagues [40]; an alternative design has been developed at Fermilab for LAr neutrino detectors

The drift field is designed to give efficient charge collection throughout the fiducial mass. Position reconstruction within the detector is expected to have a resolution of $1 \mathrm{~mm}$ in $z$ (obtained by measuring the drift time) and of $\sim 0.5 \mathrm{~cm}$ in $x$ and $y$ (obtained by fitting the spatial distribution of the $S 2$ photoelectrons detected in the top array of QUPIDS). Events from $\alpha$ decays of radioactive contaminants sitting on the side walls of the chamber will be strongly suppressed by eliminating their \$2 signals. This could be accomplished by preventing their ionization from entering the gas phase via the addition of a narrow charge collection ring to the electron extraction grid near the edge of the vessel. The $\alpha$-induced background located on the cathode will be removed by the use of the very sharp position resolution in the $z$ direction.

LIGHT YIELD: To estimate the $S 1$ light yield for DARKSIDE-50, we rely on tests performed in Princeton in 2008 and 2009. A single-phase liquid argon scintillation detector was built with a cylindrical active volume $15.2 \mathrm{~cm}$ tall and $18.5 \mathrm{~cm}$ in diameter ( $5.7 \mathrm{~kg}$ active mass). The detector volume was bounded on the side by a TPB-coated PTFE reflector and on the top and bottom by $20-\mathrm{cm}$ diameter TPB-coated acrylic windows. Two 8" Hamamatsu R5192-MOD02 PMTs, with $18 \%$ quantum efficiency, viewed the active target through the two acrylic windows. The detector was filled by passing high-purity commercial argon (99.999\% purity) through a hot $\mathrm{Zr}$-based getter [41]. This detector gave a photoelectron yield of $5 \mathrm{p} . \mathrm{e} . / \mathrm{keV}$ ec. The DARKSIDE-50 design has about the same aspect ratio between diameter and height of the DAr active volume. The fraction of the surface covered by the QUPIDS is about 75\%, with the remainder of the surface covered by a teflon reflector. This gives slightly lower photocathode coverage than in the test detector, but this is more than compensated for by the higher QUPID QE ( $\sim 35 \%$ vs. 18\% for the PMTs in the test detector) and by the presence of the teflon reflector, which has a $>90 \%$ reflectivity at $420 \mathrm{~nm}$. As a result, we believe that $>5 \mathrm{p.e.} / \mathrm{keV}_{\mathrm{ce}}$ is a conservative expectation for the S1 light yield of the DARKSIDE-50 detector.

BETA AND GAMMA BACKGROUND: Shield and detector components are selected to achieve a $\beta / \gamma$ background lower than the 10 events $/(\mathrm{kg} \cdot \mathrm{keV} \cdot \mathrm{d})$ expected from ${ }^{39} \mathrm{Ar}$ depleted by a factor $\sim 25$ relative to atmospheric argon: see Table IV. We note that with the current upper limit ${ }^{39} \mathrm{Ar}$ represents the highest source of $\beta / \gamma$ background, but it will be comparable to the cryostat and the QUPIDs if the ${ }^{39} \mathrm{Ar}$ is a factor 100 lower than the present limit, which is a possibility. We note that the tolerable $\beta / \gamma$ rate is relatively high (eight orders of magnitude above the $10^{-7}$ events $/(\mathrm{kg} \cdot \mathrm{keV} \cdot \mathrm{d}$ ) background level achieved in the Borexino fiducial volume), due to 


\begin{tabular}{|c|c|c|c|c|c|}
\hline Source & Quantity & $\begin{array}{l}{ }^{238} \mathrm{U}{ }^{232} \mathrm{Th} \\
{[\mathrm{mBq} / \mathrm{kg}]}\end{array}$ & $\begin{array}{l}\text { Cosmogenic } n \\
\text { Recoil Bkgs. } \\
{\left[(0.1 \text { ton yr })^{-1}\right]}\end{array}$ & $\begin{array}{l}\text { Radiogenic } n \\
\text { Recoil Bkgs. } \\
{\left[(0.1 \text { ton } y r)^{-1}\right]}\end{array}$ & $\begin{array}{c}\beta / \gamma \\
\text { before cuts } \\
{[\text { counts } /(\mathrm{kg} \cdot \mathrm{keV} \cdot d)]}\end{array}$ \\
\hline Scintillator [42] & $11.8 \mathrm{~T}$ & $<7.4 \times 10^{-5},<4.1 \times 10^{-6}$ & $\sim 0.12$ & $<1.5 \times 10^{-4}$ & $<2.2 \times 10^{-5}$ \\
\hline Veto PMTs [43] & 50 & $\sim 360^{*}, \sim 200^{*}$ & - & $<0.07$ & $<0.27$ \\
\hline Steel $[44,45]$ & $64.2 \mathrm{~T}$ & $<0.74,<1.1$ & $\sim 0.24$ & $<0.024$ & $<0.011$ \\
\hline Lead [46] & $119.9 \mathrm{~T}$ & $<0.01,<0.004$ & $\infty 0.72$ & $<2.2 \times 10^{-4 i}$ & $<0.001$ \\
\hline Mine Rock $[47,48]$ & - & $\sim 116,000, \sim 12,000$ & $\sim 0.51$ & $<0.01$ & $<0.003$ \\
\hline Source & Quantity & $\begin{array}{l}{ }^{238} \mathrm{U},{ }^{232} \mathrm{Th} \\
{[\mathrm{mBq} / \mathrm{kg}]}\end{array}$ & $\begin{array}{c}\text { Total } n \\
\text { production } \\
{[n / y r]}\end{array}$ & $\begin{array}{c}\text { Total } n \\
\text { Recoil Bkgs } \\
{\left[(0.1 \text { ton } \cdot y r)^{-1}\right]}\end{array}$ & $\begin{array}{c}\rho / \gamma \\
\text { before cuts } \\
[\mathrm{ev} / \mathrm{kg} \cdot \mathrm{keV} \cdot \mathrm{d})]\end{array}$ \\
\hline Acrylic Vessel $[46,49]$ & $18 \mathrm{~kg}$ & $<0.013,<0.0045$ & $<0.044$ & $<0.005$ & $<0.001$ \\
\hline Titanium Dewar $[37]$ & $73.9 \mathrm{~kg}$ & $<0.2,<0.2$ & $<4.9$ & $<0.15$ & $<0.22$ \\
\hline DAr & $50 \mathrm{~kg}$ & - & - & - & $\leq 10$ \\
\hline 3" Qupids & 38 & $<0.49^{* *},<0.40^{* *}$ & $<1.8$ & $<0.07$ & $<0.15$ \\
\hline Cu Internal Parts $[35,46]$ & $27.7 \mathrm{~kg}$ & $<0.036 .<0.0098$ & $<0.23$ & $<0.036$ & $<0.005$ \\
\hline PTFE Internal Parts [46] & $7.3 \mathrm{~kg}$ & $<0.0096 .<0.0011$ & $<0.20$ & $<0.02$ & $<1.5 \times 10^{-4}$ \\
\hline
\end{tabular}

TABLE V: Top: External Background. The expected background rates in the argon active volume arising from activity in the shield components and in the surrounding rock, as determined through Monte Carlo simulation. Note that the neutron background numbers shown do not include the application of the scintillator veto, which should remove these backgrounds with at least $99 \%$ efficiency for radiogenic neutrons and $95 \%$ efficiency for cosmogenic neutrons. The cosmogenic background rate shown is for LNGS, and (with the exception of the mine rock) includes an assumed factor of $10^{2}$ reduction from the extemal muon veto surrounding the passive shield (see text). Note that the rate of cosmogenic backgrounds would be approximately 5 times less at Sanford Lab and 70 times less at SNOLab. The $\beta / \gamma$ background are listed before any cuts (pulse shape discrimination, $\$ 2 / S 1$, multiple deposition, or scintillator veto) are applied, and includes contributions from ${ }^{40} \mathrm{~K},{ }^{60} \mathrm{Co}$ and ${ }^{137} \mathrm{Cs}$. For comparison, the depleted ${ }^{39} \mathrm{Ar}$ rate is expected to be $<10 \mathrm{ev} /(\mathrm{kg} \cdot \mathrm{keV} \cdot \mathrm{d})$. After application of the cuts, the $\beta / \gamma$ background contribute less than 0.1 events in the total exposure. Note that contamination levels achievable in the construction materials are from references cited in the first column. The level of each contaminant in the mine rock is taken to be the level of that contaminant at either SNOLab or LNGS, whichever is greater. " $\mathrm{mBq} / \mathrm{PMT}$ rather than $\mathrm{mBq} / \mathrm{kg}$. Bottom: Internal Background. Internal background sources for DARKSIDE- 50 . The neutron rates given are before the application of the scintillator veto, and include both radiogenic and cosmogenic neutrons (at the level expected at LNGS, with an assumed

$99 \%$ efficiency for the external muon veto). The $\beta / \gamma$ background are listed before any cuts (pulse shape discrimination, $S 2 / S 1$, multiple deposition cuts, or scintillator veto) and includes contributions from ${ }^{40} \mathrm{~K}$.

** $\mathrm{mBq} / \mathrm{QUPID}$ rather than $\mathrm{mBq} / \mathrm{kg}$. Beta and Gamma Background. All $\beta / \gamma$ background, internal and external, are reduced to $<0.1$ events in the 0.1 ton yr exposure by application of PSD, S2/SI discrimination, and other applicable cuts

the excellent performance of pulse shape discrimination in liquid argon. The $\beta / \gamma$ background is reduced to a negligible level, $\ll 0.1$ events in the anticipated 0.1 ton.yr exposure, after application of PSD. of the S2/S1, and other applicable discriminations, while preserving a $\sim 60 \%$ acceptance for nuclear recoils.

NEUTRON BACKGROUND: The overall neutron background budget for the detector is shown in Table IV We assumed the shallowest location (LNGS), cosmic ray flux from Ref. [50], and a muon veto efficiency of $99 \%$. Events with multiple nuclear recoils in the active mass are rejected during the analysis if any two recoils were separated by more than $0.5 \mathrm{~cm}$ vertically or $5 \mathrm{~cm}$ laterally. The simulated residual background after cuts, dominated by nuclear recoils from cosmogenic neutrons, is $<2.0$ events in the 0.1 ton yr exposure anticipated for the DARKSIDE-50 detector, before the application of the scintillator veto which is expected to further reduce the rate by a factor of 100 or more for radiogenic neutrons and 20 or more for cosmogenic neutrons.

SENSITIVITY: DARKSIDE-50 will have a $50 \mathrm{~kg}$ total target mass (see also Sec. (X). Events located within $2.5 \mathrm{~cm}$ from any edge of the active volume are removed by a fiducial cut. which leaves a fiducial mass of $33 \mathrm{~kg}$. Assuming the light yield of $>5 \mathrm{p} . \mathrm{e} . \mathrm{keV}_{\mathrm{ee}}$, the pulse shape parameters published in Ref. [51], a rejection efficiency of $\sim 100$ for the $S 2 / S 1$ discrimination, an ${ }^{39} \mathrm{Ar}$ depletion factor of 25 or larger (see Sec. V), and a nuclear quenching factor of $\sim 0.32$ [52], we calculate that the experiment will be able to set a limit, for $M_{\chi}=100 \mathrm{GeV}$, of 


\begin{tabular}{|c|c|c|c|c|}
\hline Item & Cost Item & Cost Item & Cost ltem & Cost \\
\hline Cathode HHV Supply & \$OK QUPID MHV Supplies & $\$ 37 k$ QuplDs & $\$ 250 k$ Electronics & $\$ 63 k$ \\
\hline Computer \& Storage & $\$ 14 k$ Internal Mechanics & $\$ 120 \mathrm{k}$ Ti Dewar & $\$ 100 \mathrm{k}$ Feedthroughs & $\$ 43 k$ \\
\hline Cryocooler and Controller & \$60k Cold Head & \$15k Passive Shield & $\$ 500$ k Muon Veto & $\$ 250 \mathrm{k}$ \\
\hline DAr Recovery System & \$20k Purification Unit & $\$ 20 k$ Gas Recirc. Pump & \$25k LN2 Storage Tank & $\$ 6 k$ \\
\hline Insulated Transfer Lines & $\$ 10 k$ Neutron Veto & $\$ 250$ K TPB Evaporator & $\$ 0$ Depleted Argon & $\$ 0$ \\
\hline \multirow[t]{2}{*}{ Dry Roughing Pump } & \$ok Turbo Pump & $\$ 0 k$ & & \\
\hline & & & Total & $\$ 1,783 \mathrm{k}$ \\
\hline
\end{tabular}

TABLEV: Cost of equipments for the DARKSIDE-50 detector Items listed with the cost of $\$ 0$ are independently funded.

$\sigma_{x N} \leq 1.5 \times 10^{-45} \mathrm{~cm}^{2}$ (or detect a few events/yr for a cross section of $1 \times 10^{-44} \mathrm{~cm}^{2}$ ) in a 3 yr run, corresponding to an exposure of 0.1 ton.yr after fiducialization (see Fig. 7b). We obtain consistent results using the unpublished pulse shape parameters measured in WARP-3.2 in presence of a $1 \mathrm{kV} / \mathrm{cm}$ electric field instead of those from Ref. [51].

SAFETY: A rupture disk will provide fail-safe protection of the detector against overpressure. The risk of liquid cryogen splling and asphyxiation by oxygen displacement is mitigated by the limited amount of cryogenic liquids in the detector. The choice of titanium as a construction material, the three-jacket design of the internal cryostat, and the addition of a layer of passive thermal insulator (see Sec. VIII) mitigate the risk associated with a possible mechanical failure of one of the two internal jackets and the associated possibility of a rapid phase transition of the cryogenic liquid in contact with the surrounding liquid scintillator.

PURIFICATION: The impurities of greatest concern are $\mathrm{O}_{2}, \mathrm{~N}_{2}$, and $\mathrm{H}_{2} \mathrm{O}$. Electronegative contaminants such as $\mathrm{O}_{2}$ and $\mathrm{H}_{2} \mathrm{O}$ capture electrons during drift and reduce the number surviving to the gas phase [53]. An $\mathrm{O}_{2}$ contamination $<0.3 \mathrm{ppb}$ is required to achieve an electron drift lifetime $>1 \mathrm{~ms}$ [54], as required for optimal operation of the DAr TPC. While pure DAr transmits its own scintillation light, this can be absorbed by chemical impurities. Studies by the WARP collaboration using LAr also showed significant reduction in scintillation light output in small scale detectors containing ppm levels of $\mathrm{N}_{2}$ and $\mathrm{O}_{2}$ [55].

Chemical purification of the target is expected to be well under control, due to (1) the very low temperature resulting in an extremely low out-gassing rate of $\mathrm{O}_{2}$ and $\mathrm{H}_{2} \mathrm{O}$ from surfaces and (2) data and experience from the extensive liquid argon purification R\&D performed by the ICARUS collaboration and by the FNAL liquid argon group. For example, the maximum electron drift time in the DARKSIDE-50 detector, at the baseline drift field of $800 \mathrm{~V} / \mathrm{cm}$ and with a maximum drift distance of $36 \mathrm{~cm}$, is $\sim 200 \mu \mathrm{s}$. In tests at FNAL, drift lifetimes of several milliseconds have been achieved [54] using lower performance getter systems aimed at treating much larger quantities of liquid argon.

The baseline design calls for post-distillation purification of the DAr gas by a single pass through a heated $\mathrm{Zr}$-based getter during the initial fill of the storage dewar. Heated $\mathrm{Zr}$-based getter systems capable of reducing the concentration of $\mathrm{N}_{2}, \mathrm{O}_{2}$, and $\mathrm{H}_{2} \mathrm{O}$ below $1 \mathrm{ppb}$ in noble gases are commercially available [41]. After reduction of electronegative impurities to the level of a few ppb or better during filling, experience shows that runtime purification with the same heated $Z$ r-based getters is sufficient to reduce chemical impurities in the detector liquid argon to the levels needed to obtain adequate electron drift lifetime and production and transmission of scintillation light.

ELECTRONICS: The experiment requires that the fast signals (a few ns) from the QuPIDs be read out and identified. The cathode potential $(\sim 5 \mathrm{kV})$ for the QupIos is supplied by a common channel. The Qupros require individual bias ( 400 V) for the APDs (e.g., EHS F205x_106-F [56] or SY1527 A1520P [57]) and wide bandwidth, high-gain voltage amplifiers (e.g. AU-1442-400 [58] or a modified v975 [57]). We plan to digitize the QuPID signals with a minimum vertical resolution of 12-bits and sampling frequency of $250 \mathrm{MHz}$ (e.g. $\$$ IS3350 [59] or V1720 [57]). Modern digitizers are equipped with on-board FPGAs capable of providing discrimination of the single photon signal on every channel. A conventional trigger on the $S 1$ signal (and a possible smart trigger on the $\$ 2$ signal) are then implemented by a second FPGA (e.g., PXI-7853R from National Instruments [60]).

CALIBRATIONS: The main tools for calibration will be a $d$ - $d$ neutron source (built at the Schlumberger Princeton Technology Center, on loan at the Princeton Physics Department) and a ${ }^{83 m} \mathrm{Kr}$ source (1T, $\tau=12.6 \mathrm{hr}, 18$ and $32 \mathrm{keV}$ electrons and $13 \mathrm{keV} x$-rays). The ${ }^{83 \mathrm{~m}} \mathrm{Kr}$ sources, initially developed within the KATRrN program [61], are winning rapid acceptance in the dark matter community as they provide diffuse, possibly homogeneous, sources of monoenergetic low-energy electrons [62].

RESPONSIBILITIES: The UCLA group will take responsibility for the construction of the cold head, the 


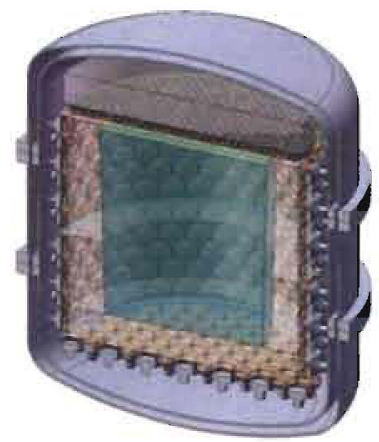

FIG. 6: The 5 ton depleted argon DarkSide detector, proposed as the next step in the DarkSide program.

cryogenic transfer lines, and the assembly of the nitrogen liquefaction systems, as well as the qualification and testing of the QUPIDS. UMass will take responsibility for the extraction grids. The Princeton group will be responsible for the collection of DAr, the assay of materials, the construction of the cryostat, the TPC and other internal mechanics of the detector, the refurbishment of the Rn-suppressed cleanroom, the construction of the neutron veto, and the integration of the entire system. The Temple University group will be responsible for the high voltage systems including simulation/design of the drift field electrodes, the HHV distribution, the feedthroughs for both drift voltage (HHV) and the QUPID acceleration voltages, and for the gas purification loop (with engineering assistance from Fermilab). The group at University of Houston will take responsibility for the procurement of the electronics and will design and implement the architecture for the DAQ and the custom firmware for the FPGA on the digitizers, in close cooperation with the group at Fermilab. The Fermilab group will take responsibility for the full simulation of the cryogenics of the detector and for the construction of the electronics trigger and the design of its firmware. The Fermilab group will also take responsibility for the passive shield and for the muon veto. The groups at Augustana College, UMass, and Princeton will lead the simulation effort. The groups at University of Virginia and at Black Hills State University obtained independent funding (NSF PHY-0903335 and NSF MR1-0923557) to develop the Cavity Ring-Down Spectroscopy trace gas analyzers for measurement of ultra-trace contaminations of $\mathrm{O}_{2}, \mathrm{~N}_{2}$, and $\mathrm{H}_{2} \mathrm{O}$. The Notre Dame group plans to pursue independent funding for the development of a quartz-lined ion chamber for ultra-trace measurement of ${ }^{39} \mathrm{Ar}$ at the ATLAS facility at Argonne National Laboratory.

STAFFING: The proposed effort, if funded at the requested level, will be supported by 24.4 FTE. For the detail of the personnel required in the University groups, see the respective Budget Justification pages. For the detail of the personnel in the Fermilab group, see the support letter from Fermilab.

MANAGEMENT: We are discussing potential candidates at Fermilab as Project Managers for DARKSIDE-50 and for the entire pre-DUSEL DarkSide program.

SCHEDULE: Our schedule calls for completion of the detector and start of commissioning in the Spring of 2011, and first detector performance data reported by the end of 2011. Initially the detector will be operated with the passive shield described above plus an inner shield of polyethylene and copper (on loan from the Princeton Plasma Physics Laboratory) replacing the neutron veto. Construction of the neutron veto will start after completing the commissioning of the DARKSIDE-50 detector in the passive shield, with its installation and commissioning foreseen for the Summer 2012.

CAPITAL PROJECT COST: All detector capital costs are listed in Table $V$ and contain a $20 \%$ contingency with the exception of the electronics and the QUPIDs where the costs are precisely known. With this proposal, we request $100 \%$ of the capital costs from the NSF. We anticipate, however, separate proposals to DOE (from UCLA, for the capital costs of the QUPIDs and electronics; from Princeton, for the capital costs of the internal mechanics) and to Fermilab/DOE (for the capital cost of the passive shield and of the muon veto). Funds requested from the NSF with this proposal may be adjusted depending on the success of other proposals to DOE.

\section{PHYSICS REACH OF DARKSIDE-50 AND FUTURE DETECTORS}

The DARKSIDE-50 detector is a small detector that will introduce new technologies to improve the sensitivity of dark matter experiments. It will also serve as a prototype for multi-ton liquid argon detectors. However, in spite 

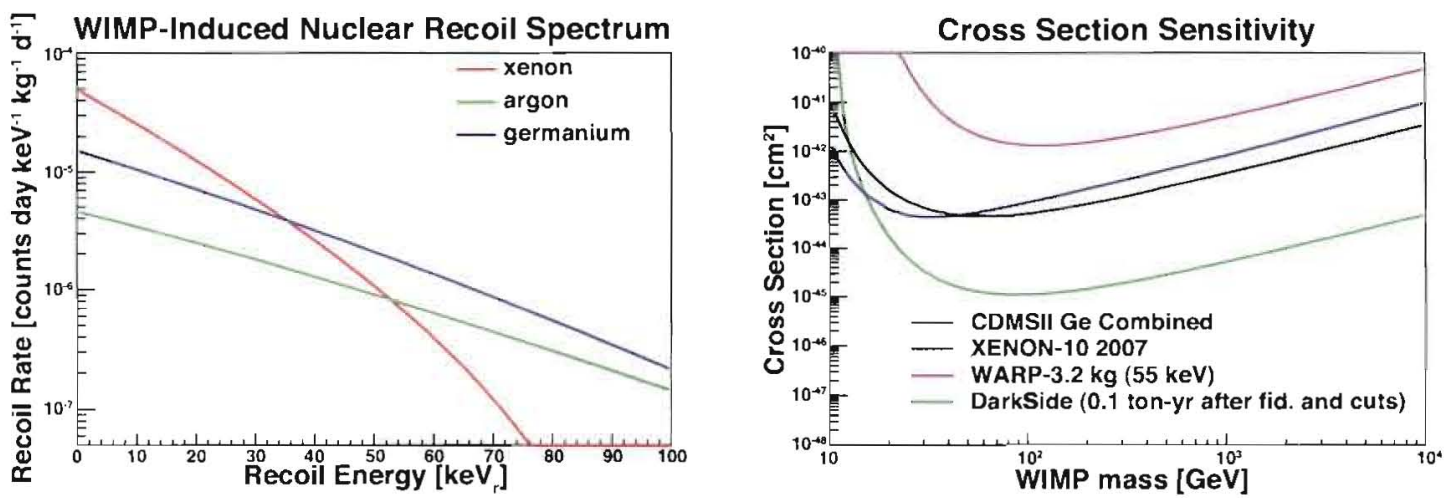

FIG. 7: (a) Nuclear recoil spectrum for $A r, G e$, and $X e$ targets $\left(M_{\chi}=100 \mathrm{GeV}\right.$ and $\left.\sigma_{\chi \mathrm{N}}=1.5 \times 10^{-45} \mathrm{~cm}^{2}\right)$. (b)

Physics reach of the DARKSIDE-50 DAr TPC ( $3 \mathrm{yr}$ run, 0.1 ton.yr exposure after fiducial and analysis cuts) compared with the limits achieved by CDMS, XENON, WARP, and ZEPLIN [4-6, 39, 63].

of its small size it will also produce significant science results. As noted in Section IX, the expected cross section limit (90\% C.L.) for spin independent WIMP scattering on argon after a background-free exposure of 0.1 ton-yr ( 3 years) is $\sim 1.5 \times 10^{-45} \mathrm{~cm}^{2}$ for a WIMP mass of $100 \mathrm{GeV}$. The exclusion limits as a function of WIMP mass that this result implies are compared with current published data in Fig. 7b. As can be noted, the improvement in sensitivity for WIMP masses of $\sim 100 \mathrm{GeV}$ is more than a factor of 10 over current published limits.

As an indication of the significance of an actual WIMP signal that might be observed, note that a cross section of $1 \times 10^{-44} \mathrm{~cm}^{2}$ would yield a count rate of a few events/yr after all nuclear recoil acceptance cuts (see Fig. $7 \mathrm{~b}$ ). A three-year background-free exposure would produce about 10 WIMP scattering events and would be strong evidence for a discovery of WIMP scattering, if the background is negligible.

If a signal is detected in DARKSIDE-50 at the level of $1 \times 10^{-44} \mathrm{~cm}^{2}$, the statistical significance of the result would be high, but the credibility of the result would depend on the measurement and understanding of backgrounds. The understanding of backgrounds, especially neutron backgrounds, which may be the most dangerous, is one of the main goals and strengths of this proposal. As noted in Sec. VI, the neutron veto detector provides measurements of radiogenic and cosmogenic neutron backgrounds that play an important role in understanding these backgrounds, possibly supporting evidence for a WIMP signal.

Other signatures available to identify a genuine spin-independent dark matter signal are (1) the $A^{2}$ factor in the nuclear cross section due to the coherent nature of the interactions [64,65], (2) the shape of the recoil energy spectrum for targets with different $A[66,67]$, (3) the annual modulation [68], and (4) the directionality of the WIMP-induced nuclear recoils [68]. The first three features can be exploited by operating of two or more detectors of the current generation.

Fig. 7a shows the recoil energy spectra for $\mathrm{Xe}$ and $\mathrm{Ar}$ with $M_{\chi}=100 \mathrm{GeV}$ and a spin independent $\sigma_{\chi N}=10^{-45} \mathrm{~cm}^{2}$. Xe is seen to have a greater sensitivity per unit mass than Ar at low energy, but Ar is less affected by the nuclear form factor correction so higher energy recoils can usefully contribute. By operating two detectors of similar sensitivity, but with targets of distinctly different atomic number, such as $\mathrm{Ar}$ and $\mathrm{Xe}$, one can provide powerful information that can be used to verify the predicted dependence on $A$ of the rate and of the recoil spectrum [69].

The DARKSIDE-50 detector will be capable of exploring other possible WIMP scenarios, including the recently suggested model of a dark matter multiplet interacting with regular matter through a light vector boson [70, 71]. DARKSIDE-50 is robustly sensitive to cross sections of $\sigma_{\chi \mathrm{N}} \approx 6 \times 10^{-45} \mathrm{~cm}^{2}$ for $M_{\chi}=100-1000 \mathrm{GeV}$ and a mass splitting between the lowest two states of the multiplet $\delta=50 \mathrm{keV}$. The ability to probe the high $\delta$ range depends strongly on the galactic escape velocity, and is significant with the widely used value $v_{\text {esc }}=600 \mathrm{~km} / \mathrm{s}$.

FUTURE PLANS: With the goal of maintaining background-free operations, we envision a 5-ton depleted argon detector as the next step in the future program, also proposed as the baseline for the MAX/Argon program at DUSEL (see Fig. 6). We note two major changes with respect to the baseline design anticipated in our S4 proposal. The possibility of developing 6" or 8" QUPIDs will benefit the program and streamline the design of the detector, eliminating the delicate acrylic vessel required for shielding neutrons from the 8" PMTs. We also anticipate that the borated liquid scintillator neutron veto will become the baseline for the next step of DARKSIDE and for the possible realization of MAX at DUSEL. We should also note that as we develop detectors for depleted 
argon, we may find that the ${ }^{39} \mathrm{Ar}$ is much lower than our current limits, which would ease our requirements for suppression of beta background.

At this time we request funding for DARKSIDE-50, a detector with a sensitivity of $1.5 \times 10^{-4.5} \mathrm{~cm}^{2}$ that also serves as a prototype for DARKSIDE 5-ton. We do not request funding of the 5-ton DARKSIDE detector, and we anticipate a separate request in 2-3 yrs, contingent on the success of the DARKSIDE-50 program.

\section{EDUCATION \& OUTREACH. BROADER IMPACT. AND PREVIOUS RESULTS}

EDUCATION \& OUTREACH: Princeton hosts a summer school for high school students from the Gran Sasso Abruzzo region of Italy [72]. The school, which was initiated by Professors Calaprice and Galbiati in 2003. brings 20-40 students each year to Princeton to study basic physics and to be exposed to the exciting scientific research that is carried out at the Gran Sasso underground laboratory. Physics classes are supplemented with lectures on topics in astrophysics. including dark matter, gravity waves, supernovae, and neutrinos. The school is open to students enroled in their fourth and fifth years in high schools of the Abruzzo region. Participants are selected on a competitive basis and are provided transportation to and from Princeton, with room and board on the Princeton University campus. Student expenses are financed by the INFN Italian science agency, the regional government of Abruzzo, and through contributions from private groups in Italy and Princeton. Researchers and faculty from Princeton and ltaly teach classes and give lectures on a volunteer basis.

With the establishment of the Sanford Laboratory and plans for DUSEL in the Homestake mine of South Dakota, the summer school was expanded in 2008 to include three students from South Dakota [73]. Following a successful first year of the joint program with Italian and American participants, the program continued in 2009 with ten American students and twenty Italian students and is set to continue in 2010. The summer school provides the students with a rich cultural experience and a unique opportunity to learn about the science and engineering focused on some of the most exciting science in modern times. It also fosters international relationships between researchers and government leaders of both regions that will benefit underground science in both countries. The American students were supported in 2009 and will be supported in 2010 and 2011 by the Davis-Bahcall Scholarships, funded by the $3 \mathrm{M}$ Corporation and intended to promote the exploration of science in South Dakota's young minds. The scholarship is named after Nobel Prize winner Dr. Ray Davis and Dr. John Bahcall, the two scientists responsible for the first exploration of solar neutrinos with the Chlorine Experiment operated in the Homestake mine.

BROADER IMPACT: The science question addressed by the DARKSIDE-50 experiment has all the ingredients to captivate the interest and imagination of young students and the general public alike. The technologies and methods that will be refined within the scope of the proposed design study will advance the application of noble liquids as imaging detectors in fields outside of particle astrophysics, including national security and medical imaging research. Equally relevant is the impact that this proposal will have in helping the visibility of DUSEL aS an international facility, recognized among a distinguished group of underground laboratories worldwide.

PREVIOUS RESULTS: The Princeton group, supported by award PHY-0503816 and PHY-0802646 completed commissioning of and operated the Borexino detector, resulting in the measurement of ${ }^{7} \mathrm{Be}$ solar neutrinos and in the first measurement of ${ }^{8} \mathrm{~B}$ solar neutrinos with a liquid scintillator target. Supported by award PHY0603376, the Princeton group took part in the WARP-3.2 kg experiment, resulting in the first dark matter search with an argon target [4] and in the WARP-140 kg detector, the largest detector for dark matter searches, currently being commissioned at LNGS. The Princeton and Notre Dame groups, supported by award PHY-0704220, conducted the study of underground argon that resulted in the discovery of sources of argon depleted in ${ }^{39} \mathrm{Ar}[9]$. Under award PHY-0811186 they will develop a facility for extraction and refinement of depleted argon. Other papers recently published by the Princeton group include Refs. [4, 8, 35, 55, 74-86]. The UCLA group was supported for its work leading to the successful operation of the first Xe-based two-phase detector, ZEPLIN-II, by awards PHY-0139065, PHY-0653459, and PHY-0810283, and for its work on the ongoing XENON-100 experiment by awards PHY 0705337 and PHY -0904224. Papers enabled by this support are Refs. [5, 69, 8793]. All groups in the collaboration are supported by award PHY -0919363 , supporting engineering and R\&D for MAX. 
[1] Committee on the Physics of the Universe, Board on Physics and Astronomy, Division on Engineering and Physical Sciences of the National Research Council of the National Academies, "Connecting Quarks with the Cosmos: Eleven Science Questions for the New Century", www nap.edu/catalog.php?record_id=10079, National Academies Press (2003).

[2] National Science and Technology Council, Committee on Science, "A 21st Century Frontier of Discovery: The Physics of the Universe", wwh ostp.gov/html/physicsoftheuriverse2. pdf (2004).

[3] The Dark Matter Scientific Assessment Group, Report on the Direct Detection and Study of Dark Matter. whrs science.doe.gov/hep/hepap_reports.shtm.

[4] P. Benetti et al. (WARP Collaboration), Astopar. Phys. 28, 495 (2008)

[5] G.J. Alner et al. (ZEPLin-11 Collaboration), Astopart. Phys. 28, 287 (2007).

[6] J. Angle et al. (Xenon Collaboration), Phys. Rev. Lett. 100, 021303 (2008).

[7] H.H. Loosli, Earth Plan. Sci Lett 63, 51 (1983).

[8] P. Benetti et al. (WARP Collaboration), Nucl. Instr. Meth. A 574, 83 (2007)

(9) D. Acosta-Kane et al. Nucl. Instr. Meth. A, 587, 46 (2008)

[10] S. Kubota, M. Hishida, and A. Nohara, Nucl. Instr. Meth. 150, 561, (1978); M.J. Carvalho and G. Klein, J. Lumin. 18-19, 487 (1979); J.W. Keto et al., J.Chem. Phys. 71,2676 (1979); T. Suemoto and H. Kanzaki, J. Phys. Soc. Japan 46, 1554 (1979); S. Kubota et al., Nucl. Instr. Meth. 196, 101 (1982); S. Kubota et al., Phys. Rev. B 20, 3486 (1979).

[11] E. Shibamura, K. Masuda, and T. Doke, Research Report to the Ministry of Education, Science, Sport, and Culture for Grant-in-Aid Scientific Research(C). No. 62540284 (1988).

[12] P. Benetti et al, Nucl. Instr. Meth. A 327, 203 (1993).

[13] A. Hitachi et al., Phys. Rev. B 27, 5279 (1983).

[14] M.G. Boulay and A. Hime, Astropart. Phys. 25, 179 (2006).

[15] L. Grandi, University of Pavia, Doctoral Thesis, pages 178-179(2005).

[16] C. Jillings for the DEAP/CLEAN Collaboration, Report to SNOLAB2009, Talk at the SNOLab Worskhop 2009, Lively, ON (2009). Slides available at snolab2009.snolab.ca/progran.btml.

[17] K. Arisaka, Nucl. Instrum. Meth. A 442, 80 (2000)

[18] K. Arisaka, "Summary Talk", 4th International Conference on New Developments in Photodetection (Beaune 05), Beaune, France, 19.24 Jun 2005

[19] W. Wilson, R. Perry, W. Charton, T. Parish, G. Estes, T. Brown, E. Arthur, M. Bozolan, T. England, D. Madland, et al., SOURCES 4A: A Code for Calculating $(\alpha, n)$ Spontaneous Fission, and Delayed Neutron Sources and Spectra LA-13639-MS. LANL (1999).

[20] Ph. Collon, F. Calaprice, C. Gabiati, and S. Mukhopadhyay, Study of Argon for WMP Dark Matter Detectors and Earth Sciences, Proposal to NSF, Princeton University (2006).

[21] F. Calaprice, C Galbiati, and P. Meyers, DUSEL R\&D Depleted Argon from Underground Sources, Proposal to NSF, Princeton University (2007).

[22] A. Sonnenschein et al., DUSEL R\&D Proposal: Measurement of Ar-39 in Argon, FNAL (2007).

[23] R.S. Raghavan and S. Pakvasa, Phys. Rev, D 37, 849 (1988).

24] G. Alimonti et al. (Borexino Collaboration), Astropart. Phys. 16, 205 (2002).

[25] Yi-Fen Yen et al., Nucl. Instr. Meth. A 447, 476 (2000).

[26] L. Swiderski et al, Nucl. Sci. Symp. Conf. Rec, 1389 (2007).

[27] L. Swiderski et al., Nucl. Sci. Symp. Conf. Rec., 2378 (2008).

[28] M. Flaska and S.A. Pozzi, Nuct Instr. Meth. A 599, 221 (2009)

[29] See the brochuers at www saintgobain. com.

[30] F. Arneodo et al. (ICARUS Collaboration). Nucl. Instr. Meth. A 418, 285 (1998),

[31] L.R. Greenwood and N.R. Chellew, Rev. Sci. Instrum. 50, 466 (1979).

[32] D.S. Akerib et al. (CDMS Collaboration), Phys. Rev. D 72, 052009 (2005).

[33] R. Heaton. H. Lee, P. Skensved, and B. Robertson. Nucl. Instr. Meth. A 276, 529 (1989).

[34] A. Pocar, Low Background Techniques and Experimental Challenges for Borexino and its Nylon Vessels, Ph.D. Dissertation. Princeton University (2003).

[35] 1. Benziger et al., Nud. Instr. Meth. A 582, 509 (2007).

[36] B. Aharmim et al. (SNO Collaboration). Phys. Rev. Lett. 101, 111301 (2008).

[37] D. Mckinsey for the LUX Collaboration, The LUX Dark Matter Experiment, Talk at the TAUP 2009 Conference, Rome, Italy (2009). Slides available at taup2009. Ings. infn.it/slides/jul1/mckinsey.pdf.

[38] T. Iwamoto et al., Development of a large volume zero boil-off liquid xenon storage system for muon rare decay experiment (MEG), in press on Cryogenics (2009)

[39] V. Lebedenko et al. (ZEPLN-IIl collaboration), to be published in Astropar. Phys. (2009).

[40] S. Amerio et al. (ICARUS Collaboration), Nucl. Instr. Meth. A 527, 329 (2004) 
[41] See the brochure of the SAES Getters PS5 Series at saespuregas. com.

[42] G. Bellini et al. (Borexino Collaboration), Borexino at Gran Sasso, Proposal for a Real Time Detector for Low Energy Solar Neutrinos, Proposal to INFN (1991).

[43] P. Mosteiro, Evaluation of neutron background from PMTs in dark matter detectors, Experimental Project, Princeton University (2008).

[44] E. de Haas and F. Calaprice, private communication.

[45] C. Arpesella et al. (Borexino Collaboration), Astropart. Phys. 18, 1 (2002).

[46] D.S. Leonard et al., Nucl. Instr. Meth. A 591, 490 (2008).

[47] C. Arpesella, Appl. Radiat. Isot. 47, 991 (1996).

[48] SNOLAB Users Handbook, SNOLab (2006). Available at snolab2008. snolab.ca/snolab_users_handbook_rev02. pdf.

[49] J. Boger et al. (SNO Collaboration), Nucl. Instr. Meth. A 449, 172 (2000).

[50] D.-M. Mei and A. Hime, Phys. Rev. D 73, 053004 (2006); D.-M. Mei, Z.-B. Yin, and S.R. Elliott, Astropart. Phys. 31, 417 (2009).

[51] H. Lippincott et al., Phys. Rev C 78, 035801 (2008).

[52] J. Nikkel for the DEAP/CLEAN Collaboration, Development of the MiniCLEAN dark matter detector, Talk at the TAP 2009 Conference, Roma, Italy (2009). Slides available at taup2009. Ings.infn.it/parallel_1.html.

[53] G. Bakale, U. Sowada, and W.F. Schmidt, J. Phys. Chem. 80, 2556 (1976).

[54] P. Cennini et al. (ICARUS Collaboration), Nucl. Inst. Meth. A 333, 567 (1993); D. Finley et al., "Work at FNAL to achieve long electron drift lifetime in liquid argon", FERMILAB-TM-2385-E (2006).

[55] R. Acciarri et al. (WARP Collaboration), Nucl. Instr. Meth. A 607 (2009) 169.

[56] See brochures at www.wiener.de

[57] See brochures at www.caen. it

[58] See brochures at www.miteq. com

[59] See brochures at www. struck.de

[60] See brochures at www.ni.com

[61] D. Vénos, A. Špalek, O. Lebeda, M. Fišer, Appl. Rad. Isotopes 63, 323 (2005); D. Vénos, O. Dragoun, A. Špalek, and M. Vobecký, Nucl. Instr. Meth. A 560, 352 (2006).

[62] L.W. Kastens, S.B. Cahn, A. Manzur, and D.N. McKinsey, Calibration of a Liquid Xenon Detector with ${ }^{83 m}$ Kr. arXiv:0905.1766v1 (2009).

[63] Z. Ahmed et al. (CDMS Collaboration), Phys. Rev. Lett. 102, 011301 (2009)

[64] A. Drukier and L. Stodolsky, Phys. Rev. D 30, 2295 (1984).

[65] M.W. Goodman and E. Witten, Phys. Rev. D 31, 3059 (1985).

[66] G. Jungman, M. Kamionkowski, and K. Griest, Phys. Rep. 267, 195 (1996).

[67] P.F. Smith and J.D. Lewin, Phys. Rep. 187, 203 (1990); J.D. Lewin and P.F. Smith, Astropart. Phys. 6, 87 (1996).

[68] D.N. Spergel, Phys. Rev. D 37, 1353 (1988).

[69] K. Arisaka et al., Astropart. Phys. 31, 63 (2009).

[70] N. Arkani-Hamed, D.P. Finkbeiner, T. Slatyer, and N. Weiner, Phys. Rev. D 79, 015014 (2009).

[71] D. Smith and N. Weiner, Phys. Rev. D 64, 043502 (2001); D. Tucker-Smith and N. Weiner, Phys. Rev. D 72, 063509 (2005); S. Chang, G.D. Kribs, D. Tucker-Smith, and N. Weiner, Phys. Rev. D 79, 043513 (2009).

[72] Website of the Gran Sasso-Princeton-South Dakota Physics Summer School, www.physics.princeton.edu/www/ $\mathrm{jh} /$ gransasso/.

[73] Website of the David-Bahcall Scholarship, www. SummerScience2009.com.

[74] H.O. Back et al. (Borexino Collaboration), Response to the critics of Borexino result in "A new experimental limit for the stability of the electron" by H.V. Klapdor-Kleingrothaus, I.V. Krivosheina, and I.V. Titkova, submitted to Astrop. Phys., arXiv:hep-ex/0703044 (2007).

[75] H.O. Back et al. (Borexino Collaboration), Nucl. Instr. Meth. A 584, 98 (2008).

[76] C. Arpesella et al., (Borexino Collaboration), Phys. Lett. B 658, 101 (2008).

[77] H.O. Back et al. (Borexino Collaboration), Nucl. Instr. Meth. A 585, 48 (2008).

[78] G. Bellini et al. (Borexino Collaboration), Eur. Phys. J. C 54, 61 (2008).

[79] J. Benziger et al., Nucl. Instr. Meth. A 587, 277 (2008).

[80] C. Arpesella et al. (Borexino Collaboration), Phys. Rev. Lett 101, 091302 (2008).

[81] G. Bellini et al. (Borexino Collaboration), Measurement of the solar ${ }^{8} B$ neutrino flux with 246 live days of Borexino and observation of the MSW vacuum-matter transition, arXiv:0808.2868 (2008).

[82] J. Benziger et al., Nucl. Instr. Meth. A 600, 58 (2009).

[83] J. Benziger et al., Nucl. Instr. Meth. A 608, 464 (2009).

[84] G. Alimonti et al. (Borexino Collaboration), Nucl. Instr. Meth. A 609, 58 (2009).

[85] A.A. Aguilar-Arevalo et al., (MiniBooNE Collaboration), Phys. Rev. Lett. 98, 231801 (2007).

[86] A.A. Aguilar-Arevalo et al., (MiniBoonE Collaboration), Nucl Instr. Meth. A 599, 28 (2009)

[87] T.J. Sumner et al. (ZEPLIN Collaboration), Nucl. Phys. B (Proc. Suppl.) 173, 108 (2007).

[88] R. Bisset et al. (ZEPLIN Collaboration), Nucl. Phys. B (Proc. Suppl.) 173, 164 (2007). 
[89] D. Akimov et al. (ZEPLIN Collaboration), Astropart. Phys. 27, 46 (2007).

[90] G. Alner et al. (ZEPLIN Collaboration). Phys. Lett. B 653, 161 (2007).

[91] G.J. Alner et al. (ZEPLIN Collaboration), Nucl. Instr. Meth. A 587, 101 (2008)

[92] H.M. Araújo et al (ZEPLIN Collaboration). Astropart. Physics 29471 (2008)

[93] B. Edwards et al. (ZEPLIN Collaboration), Astropart. Phys. 30, 54 (2008). 